\title{
Equivalence of two approaches to study the stress-strain relation in the myocardium
}

\author{
R. M. Shoucri \\ Department of Mathematics and Computer Science, \\ Royal Military College of Canada, Kingston, Ontario, Canada
}

\begin{abstract}
A method to study the mechanics of ventricular contraction was developed in previous publications by the author. In those studies, the active force of the myocardium is represented as force per unit volume of the myocardium. Other authors have developed studies in which the active force of the myocardium is included in the expression of the total stress derived from the constitutive relations. The purpose of the present study is to show how to make the connection between these two approaches. Derivation is done in a general way, expressions for the stress components are derived and application to experimental data is presented. The possibility of relating the pseudo strain energy function to the tension generated by the muscular fibre is also shown.
\end{abstract}

Keywords: cardiac mechanics, mathematical modelling of ventricular contraction, pressure-volume relation, active force of the myocardium, pseudo strain energy function.

\section{Introduction}

In previous studies by the author a method to study the stress-strain relation in the myocardium was developed in which the active force generated by the myocardium was represented as force per unit volume of the myocardium [1-6]. This mathematical approach used a cylindrical model of the left ventricle and was successfully developed by using both large elastic deformation $[1,2]$ and linear elasticity $[3,4]$, the transition from large elastic deformation to linear elasticity was discussed in [6]. Most other studies that have been developed focus on the way the expression of the total stress can be derived from the constitutive relations [9-11]. 
In this study the myocardium is represented as a thick-walled incompressible cylinder in which the myocardial fibres are imbedded in a helical way in a soft incompressible medium (see fig. 1). The contraction of the cylinder is modelled is a way to take into consideration torsion and shear, it turns out that their effect is small for the purpose of the numerical calculation of this study. In the quasistatic approximation used in this study, inertia forces and viscous forces are neglected. The total stress induced in the passive medium of the myocardium $t_{i j}$ is written in the form $t_{i j}=\sigma_{i j}+q_{i j}$, where $q_{i j}$ is the stress induced by the muscular fibre tension $T$ and reflects the directional character of the stress; $\sigma_{i j}$ is the stress resulting from the deformation of the passive medium of the myocardium (passive medium assumed isotropic). A similar approach can de found in Spencer [11]. Nevo and Lanir [8] have introduced a quantity similar to $q_{i j}$ as the derivative of a hydrostatic pressure, and Arts et al. [12] have used an approach where $\sigma_{i j}$ is replaced by a hydrostatic pressure.

The purpose of this study is to show the equivalence of the formalism developed in [1-6] by the author with the formalism developed by Humphrey and Yin [9] in which the total stress $t_{i j}$ induced in the myocardium is derived from a pseudo strain energy function $W$. It is also shown how $W$ can be directly related to the muscular fibre tension $T$, and that the splitting of $W=W_{\text {iso }}+W_{\text {aniso }}$ into an isotropic and an anisotropic component $[9,13,14]$ is equivalent to the aforementioned splitting of the total stress $t_{i j}$.

\section{Mathematical formalism: first approach}

\subsection{Equilibrium conditions}

This is the approach used in [9, 10], in which the calculation is carried out by using the total stress $t_{i j}$. By assuming symmetry around the $\mathrm{z}$-axis (solution independent of $\theta$ ), the conditions of local equilibrium the myocardium $(\operatorname{div} \boldsymbol{t}=0)$ can be written as follows in cylindrical coordinates

$$
\begin{gathered}
\frac{\partial t_{r r}}{\partial r}+\frac{t_{r r}-t_{\theta \theta}}{r}+\frac{\partial t_{z r}}{\partial z}=0 \\
\frac{1}{r^{2}} \frac{\partial\left(r^{2} t_{r \theta}\right)}{\partial r}+\frac{\partial t_{z \theta}}{\partial z}=0 \\
\frac{1}{r} \frac{\partial\left(r t_{r z}\right)}{\partial r}+\frac{\partial t_{z z}}{\partial z}=0
\end{gathered}
$$

The stress can be dependent on the $z$ variable, but we shall simplify the mathematical formalism by assuming that $t_{z r}, t_{z \theta}$ and $t_{z z}$ are independent of $z$ as in [9]. In this case eqns (1b) and (1c) give 


$$
r^{2} t_{r \theta}=\text { const }=H_{1} \quad r t_{r z}=\text { const }=H_{2}
$$

The radial stress boundary conditions on the surface of the cylinder are given by (see fig. 1).

$$
t_{r r}\left(r_{i}\right)=-P_{i} \quad t_{r r}\left(r_{o}\right)=-P_{o}
$$

\subsection{Deformation gradient}

The contraction of the myocardium is assumed to change the stress free configuration $(R, \Theta, Z)$ to the end-diastolic configuration $\left(r_{e d}, \theta_{e d}, z_{e d}\right)$ and finally to $(r, \theta, z)$ during the systolic phase according to the relations

$$
\begin{gathered}
r_{e d}=r_{e d}(R), \quad \theta_{e d}=\alpha_{1} \Theta, \quad z_{e d}=k_{1} Z \\
r=r\left(r_{e d}\right), \quad \theta=\alpha_{2} \theta_{e d}+\psi_{2} z_{e d}+\chi\left(r_{e d}\right), \\
z=k_{2 z} z_{e d}+k_{2 \theta} \theta_{e d}+\omega\left(r_{e d}\right)
\end{gathered}
$$

which combined together give

$$
r=r(R), \quad \theta=\alpha \Theta+\psi Z+\chi(R), \quad z=k_{z} Z+k_{\theta} \Theta+\omega(R)
$$

The deformation gradient $\boldsymbol{F}_{\boldsymbol{1}}$ for the transformation from the stress free configuration $(R, \Theta, Z)$ to the end-diastolic configuration $\left(r_{e d}, \theta_{e d}, z_{e d}\right)$ in cylindrical coordinates is given by [10]

$$
F_{1}=\left[\begin{array}{ccc}
\frac{\partial r_{e d}}{\partial R} & \frac{1}{R} \frac{\partial r_{e d}}{\partial \Theta} & \frac{\partial r_{e d}}{\partial Z} \\
r_{e d} \frac{\partial \theta_{e d}}{\partial R} & \frac{r_{e d}}{R} \frac{\partial \theta_{e d}}{\partial \Theta} & r_{e d} \frac{\partial \theta_{e d}}{\partial Z} \\
\frac{\partial z_{e d}}{\partial R} & \frac{1}{R} \frac{\partial z_{e d}}{\partial \Theta} & \frac{\partial z_{e d}}{\partial Z}
\end{array}\right]=\left[\begin{array}{ccc}
\frac{d r_{e d}}{d R} & 0 & 0 \\
0 & \alpha_{1} \frac{r_{e d}}{R} & 0 \\
0 & 0 & k_{1}
\end{array}\right]
$$


The deformation gradient $\boldsymbol{F}_{2}$ for the transformation from the end-diastolic configuration $\left(r_{e d}, \theta_{e d}, z_{e d}\right)$ to the final configuration $(r, \theta, z)$ is given by

$$
F_{2}=\left[\begin{array}{cccc}
\frac{\partial r}{\partial r_{r e}} & \frac{1}{r_{e d}} \frac{\partial r}{\partial \theta_{e d}} & \frac{\partial r}{\partial z_{e d}} \\
r \frac{\partial \theta}{\partial r_{e d}} & \frac{r}{r_{e d}} \frac{\partial \theta}{\partial \theta_{e d}} & r \frac{\partial \theta}{\partial z_{e d}} \\
\frac{\partial z}{\partial r_{e d}} & \frac{1}{r_{e d}} \frac{\partial z}{\partial \theta_{e d}} & \frac{\partial z}{\partial z_{e d}}
\end{array}\right]=\left[\begin{array}{ccc}
\frac{d r}{d r_{e d}} & 0 & 0 \\
r \frac{d \chi}{d r_{e d}} & \alpha_{2} \frac{r}{r_{e d}} & r \psi_{2} \\
\frac{d \omega}{d r_{e d}} & \frac{k_{2 \theta}}{r_{e d}} & k_{2 z}
\end{array}\right]
$$
by

The deformation gradient $\boldsymbol{F}=\boldsymbol{F}_{2} \cdot \boldsymbol{F}_{\boldsymbol{1}}$ of the combined transformation is given

$$
F=\left[\begin{array}{ccc}
\frac{\partial r}{\partial R} & \frac{1}{R} \frac{\partial r}{\partial \Theta} & \frac{\partial r}{\partial Z} \\
r \frac{\partial \theta}{\partial R} & \frac{r}{R} \frac{\partial \theta}{\partial \Theta} & r \frac{\partial \theta}{\partial Z} \\
\frac{\partial z}{\partial Z} & \frac{1}{R} \frac{\partial z}{\partial \Theta} & \frac{\partial z}{\partial Z}
\end{array}\right]=\left[\begin{array}{ccc}
\frac{d r}{d R} & 0 & 0 \\
r \frac{d \chi}{d R} & \alpha \frac{r}{R} & r \psi \\
\frac{d \omega}{d R} & \frac{k_{\theta}}{R} & k_{z}
\end{array}\right]
$$

where $\alpha=\alpha_{2} \alpha_{1}, \psi=\psi_{2} k_{l}, k_{\theta}=k_{2 \theta} \alpha_{l}, k_{z}=k_{2 z} k_{l}$. By assuming that the transformations take place at constant volume, the incompressibility constraint can be written as follows

$$
I_{3}=(\operatorname{det} F)^{2}=\left(\operatorname{det} F_{2}\right)^{2}\left(\operatorname{det} F_{1}\right)^{2}=1
$$

where $I_{3}$ is the third strain invariant. By calculating the determinants in eqn (10) one obtains

$$
\frac{d r}{d R}=\frac{R}{K r}, \quad K r^{2}-R^{2}=K r_{i}^{2}-R_{i}^{2}
$$




$$
\begin{array}{cc}
\frac{d r_{e d}}{d R}=\frac{R}{K_{1} r_{e d}}, & K_{1} r_{e d}^{2}-R^{2}=K_{1} r_{i e d}^{2}-R_{i}^{2} \\
\frac{d r}{d r_{e d}}=\frac{r_{e d}}{K_{2} r}, & K_{2} r^{2}-r_{e d}^{2}=K_{2} r_{i}^{2}-r_{\text {ied }}^{2}
\end{array}
$$

We have $K_{1}=\alpha_{1} k_{1}=\operatorname{det}\left(F_{1}\right), K_{2}=\alpha_{2} k_{2 z}-\psi_{2} k_{2 \theta}=\operatorname{det}\left(F_{2}\right)$ and $K=K_{1} K_{2}=$ $\operatorname{det}(F)=\alpha_{1} k_{1} \alpha_{2} k_{2 z}-\alpha_{1} k_{2} k_{l} \psi_{2}=\alpha k_{z}-k_{\theta} \psi$, with $\alpha=\alpha_{1} \alpha_{2}, k_{z}=k_{1} k_{2 z}, \psi=\psi_{2} k_{1}$, $k_{\theta}=\alpha_{1} k_{2 \theta}$. The inner radii are respectively $R_{i}$ and $r_{i}$ in the stress free configuration and during the systolic phase.

A muscular fibre in the myocardium is supposed to have a helical form on a cylindrical surface. In the undeformed configuration a unit vector $\boldsymbol{N}$ with fibre angle $\Gamma(R)$ is transformed into a vector $\boldsymbol{n}$ in the deformed configuration with fibre angle $\gamma(r)$ calculated with respect to the circumferential direction. With $\lambda_{N}$ representing the stretch ratio in the direction of the muscular fibre, we have

$$
\boldsymbol{n}=\left[0, \cos \left(\gamma(r), \sin (\gamma(r)]^{T}, \quad \boldsymbol{N}=\left[0, \cos \left(\Gamma(R), \sin (\Gamma(R)]^{T}\right.\right.\right.\right.
$$

with

$$
\boldsymbol{n}=\frac{1}{\lambda_{N}} \boldsymbol{F} . \boldsymbol{N}
$$

By using eqns (9) and (15) we get

$$
\begin{aligned}
& \cos (\gamma(r))=\frac{1}{\lambda_{N}}\left[\frac{\alpha r}{R} \cos (\Gamma(R))+(r \psi) \sin (\Gamma(R))\right] \\
& \sin (\gamma(r))=\frac{1}{\lambda_{N}}\left[\frac{k_{\theta}}{R} \cos (\Gamma(R))+k_{z} \sin (\Gamma(R))\right]
\end{aligned}
$$

\subsection{Constitutive relations}

Relations between the components of the stress and deformation are known as constitutive relations. By assuming transverse isotropy with respect to the z-axis of the cylinder, Humphrey and Yin [9] have focused on a subclass of transverse isotropic material with pseudo strain energy function $W$ given by the expression

$$
W=W\left(I_{1}, \lambda_{N}\right)
$$

where $I_{l}$ is the first strain invariant $I_{l}=\operatorname{tr}(\boldsymbol{B})$, and $\boldsymbol{B}=\boldsymbol{F} . \boldsymbol{F}^{\boldsymbol{T}}$ is the left CauchyGreen deformation tensor. Written explicitly we have 


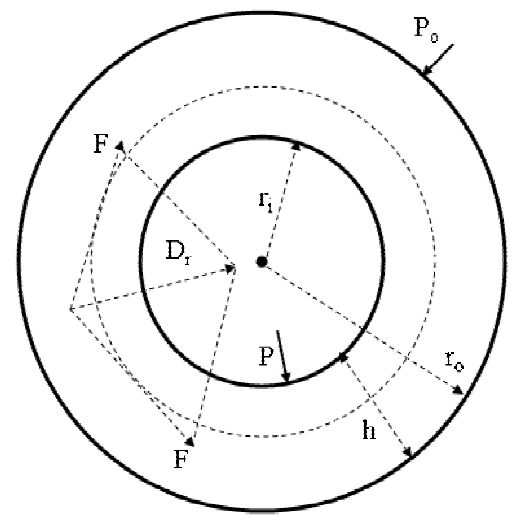

Figure 1: Cross-section of a cylinder representing the left ventricle. The dotted circle represents the projection of a helical fibre on the cross-section. $D_{r}(r)$ is the radial active force/unit volume of the myocardium. $P_{i}$ is the ventricular pressure, $P_{o}$ is the outer pressure, $r_{i}$ is the inner radius, $r_{o}$ is the outer radius, $h=b-a$ is the thickness of the myocardium.

$$
B=\left[\begin{array}{ccc}
\left(\frac{d r}{d R}\right)^{2} & r \frac{d r}{d R} \frac{d \chi}{d R} & \frac{d r}{d R} \frac{d \omega}{d R} \\
r \frac{d r}{d R} \frac{d \chi}{d R} & \left(r \frac{d \chi}{d R}\right)^{2}+\frac{\alpha^{2} r^{2}}{R^{2}}+\left(r \psi^{2}\right) & r \frac{d \chi}{d R} \frac{d \omega}{d R}+\frac{\alpha r k_{\theta}}{R^{2}}+r \psi k_{z} \\
\frac{d r}{d R} \frac{d \omega}{d R} & r \frac{d \chi}{d R} \frac{d \omega}{d R}+\frac{\alpha r k_{\theta}}{R^{2}}+r \psi k_{z} & \left(\frac{d \omega}{d R}\right)^{2}+\frac{k_{\theta}^{2}}{R^{2}}+k_{z}^{2}
\end{array}\right]
$$

The Cauchy stress $\boldsymbol{t}$ (force/current area) can be expressed by using eqn (18) in the form [9]

$$
t_{i j}=-p \delta_{i j}+2 W_{1} B_{i j}+W_{\lambda N} \lambda_{N} n_{i} n_{j}
$$

where $W_{1}=\frac{\partial W}{\partial I_{1}}$ and $W_{\lambda N}=\frac{\partial W}{\partial \lambda_{N}}, p$ is a Lagrange multiplier introduced to express the incompressibility condition for the myocardium. The first two terms in eqn (20) have an isotropic symmetry and the third term (the components of $n_{i}$ are shown in eqn (14), part one) has a directional character corresponding to the 
direction of the muscular fibre in the myocardium. We are now in a position to make the junction with the second approach developed in [1-6].

\section{Mathematical formalism: second approach}

\subsection{Equilibrium conditions}

We shall now see that it can be more instructive to work with the components $\sigma_{i j}$ and $q_{i j}$ of $t_{i i}=\sigma_{i j}+q_{i j} . T(r, z)$ is the stress in the direction of the muscular fibre in the myocardium. By assuming that a muscular fibre in the myocardium has a helical cylindrical shape as in the previous section, one can derive for the components of the stress $q_{i j}$ the following relations as in [2, 15]

$$
\begin{gathered}
q_{r r}=0, \quad q_{\theta \theta}=T(\cos \gamma(r))^{2}, \quad q_{z z}=T(\sin \gamma(r))^{2} \\
q_{z \theta}=T \sin \gamma(r) \cos \gamma(r), \quad q_{r \theta}=q_{r z}=0
\end{gathered}
$$

It is also possible to write the following relations

$$
D_{r}=\frac{T}{r}(\cos \gamma(r))^{2}, \quad q_{z \theta}=r D_{r} \tan \gamma, \quad q_{z z}=r D_{r} \tan ^{2} \gamma
$$

where $D_{r}$ is obtained by substituting $t_{i j}=\sigma_{i j}+q_{i j}$ in eqns (1) and by writing the terms containing $q_{i j}$ in the following way

$$
D_{r}=\frac{q_{\theta \theta}}{r}, \quad D_{\theta}=-\frac{\partial q_{z \theta}}{\partial z}, \quad D_{z}=-\frac{\partial q_{z z}}{\partial z}
$$

The quantities $D_{r}, D_{\theta}$ and $D_{z}$ have the units of force/unit volume of the myocardium expressed in the three orthogonal directions of a cylindrical coordinate system. By using this notation, eqns (1) can be written in the form

$$
\begin{gathered}
\frac{\partial \sigma_{r r}}{\partial r}+\frac{\sigma_{r r}-\sigma_{\theta \theta}}{r}+\frac{\partial \sigma_{z r}}{\partial z}-D_{r}=\frac{\partial \sigma_{r r}}{\partial r}+\frac{\sigma_{r r}-\sigma_{\theta \theta}}{r}+\frac{\partial}{\partial z}\left(\sigma_{z r}-\int_{z} D_{r} d z\right) \\
\frac{1}{r^{2}} \frac{\partial\left(r^{2} \sigma_{r \theta}\right)}{\partial r}+\frac{\partial \sigma_{z \theta}}{\partial z}-D_{\theta}=\frac{1}{r^{2}} \frac{\partial\left(r^{2} \sigma_{r \theta}\right)}{\partial r}+\frac{\partial}{\partial z}\left(\sigma_{z \theta}-\int_{z} D_{\theta} d z\right) \\
\frac{1}{r} \frac{\partial\left(r \sigma_{r z}\right)}{\partial r}+\frac{\partial \sigma_{z z}}{\partial z}-D_{z}=\frac{1}{r} \frac{\partial\left(r \sigma_{r z}\right)}{\partial r}+\frac{\partial}{\partial z}\left(\sigma_{z z}-\int_{z} D_{z} d z\right)
\end{gathered}
$$


Because $t_{r \theta}=\sigma_{r \theta}\left(q_{r \theta}=0\right)$ and $t_{r z}=\sigma_{r z}\left(q_{r z}=0\right)$ we can write in a way similar to eqns (2)

$$
r^{2} \sigma_{r \theta}=\text { const }=H_{1} \quad r \sigma_{r z}=\text { const }=H_{2}
$$

We assume that no external moment is applied to the myocardium, consequently the moment of forces $M$ around the z-axis is zero

$$
M=2 \pi \int_{r i}^{r o}\left(\sigma_{z \theta}+q_{z \theta}\right) r^{2} d r=0
$$

which gives

$$
\sigma_{z \theta}=-q_{z \theta}
$$

Equilibrium of forces in the longitudinal direction gives

$$
\sigma_{z z}+q_{z z}+\tau_{a v}=0
$$

where $\tau_{a v}$ is the average traction on the cross-section and is given by [8]

$$
\tau_{a v}=\frac{P_{i} r_{i}^{2}-P_{o} r_{o}^{2}}{r_{o}^{2}-r_{i}^{2}}
$$

It is assumed that the average stress $\tau_{a v}$ is independent of $r$ and $z$.

\subsection{Constitutive relations}

It is assumed that the muscular fibre tension $T(r, z)$, and consequently $q_{i j}$, is uniformly distributed throughout the myocardium. By writing $t_{i j}=\sigma_{i j}+q_{i j}$ and by comparing eqns (21) with the last term of eqn (20) we can write

$$
q_{i j}=W_{\lambda N} \lambda_{N} n_{i} n_{j}
$$

with $W_{\lambda N}$ appropriately chosen such that

$$
T=W_{\lambda N} \lambda_{N}
$$

From eqn (20), the stress $\sigma_{i j}$ can be expressed as follows

$$
\sigma_{i j}=-p \delta_{i j}+2 W_{1} B_{i j}
$$


This last equation can be written with the help of eqn (19) in explicit form as follows

$$
\begin{gathered}
\sigma_{r \theta}=2 W_{1} r \frac{d r}{d R} \frac{d \chi}{d R}=\frac{H_{1}}{r^{2}} \\
\sigma_{r z}=2 W_{1} \frac{d r}{d R} \frac{d \omega}{d R}=\frac{H_{2}}{r} \\
\sigma_{z \theta}=2 W_{1}\left[r \frac{d \chi}{d r} \frac{d \omega}{d R}+\frac{\alpha r k_{\theta}}{R^{2}}+r \psi k_{z}\right] \\
\sigma_{z z}=-p+2 W_{1}\left[\left(\frac{d \omega}{d r}\right)^{2}+\frac{k_{\theta}^{2}}{R^{2}}+k_{z}^{2}\right] \\
\sigma_{\theta \theta}=-p+2 W_{1}\left[\left(r \frac{d \chi}{d r}\right)^{2}+\left(\frac{\alpha r}{R}\right)^{2}+(r \psi)^{2}\right] \\
\sigma_{r r}=-p+2 W_{1}\left(\frac{d r}{d R}\right)^{2}=-p+2 W_{1}\left(\frac{R}{K r}\right)^{2}
\end{gathered}
$$

These equations are used in the experimental application described in what follows. The term $D_{r}$ appearing in eqn (24a) is similar, but not identical, to the introduction of a derivative of a hydrostatic pressure in eqn (26) of [8].

\section{Application and results}

The fibre angle $\gamma(r)$ (referred to the circumferential direction) is supposed to be constant with respect to the axial and circumferential directions. The radial variation of the fibre angle is supposed to be linear and given by

$$
\gamma=\gamma_{\text {end }}\left(\frac{r_{o}-r}{r_{o}-r_{i}}\right)+\gamma_{\text {epi }}\left(\frac{r-r_{i}}{r_{o}-r_{i}}\right)
$$

where $\gamma_{\text {end }}=45^{\circ}$ is the fibre angle at the endocardium, and $\gamma_{\text {epi }}=-45^{\circ}$ is the fibre angle at the epicardium. The dimensions of the left ventricle in the diastolic configuration are outer radius $r_{o}=3.38 \mathrm{~cm}$, inner radius $r_{i}=1.02 \mathrm{~cm}$ and length $\digamma=3.06 \mathrm{~cm}$ as taken from experiment on dog reported in Feit [7]. The corresponding radii in the reference stress free configuration were estimated from eqn (11), part two, as $R_{o}=3.4474 \mathrm{~cm}$ and $R_{i}=1.1 \mathrm{~cm}$. The tension $T$ developed by the muscular by the fibre near the end-diastolic phase is taken from fig. 7a of Feit [7] and is reproduced in fig. 2 (left) of this study, fig. 2 (right) 

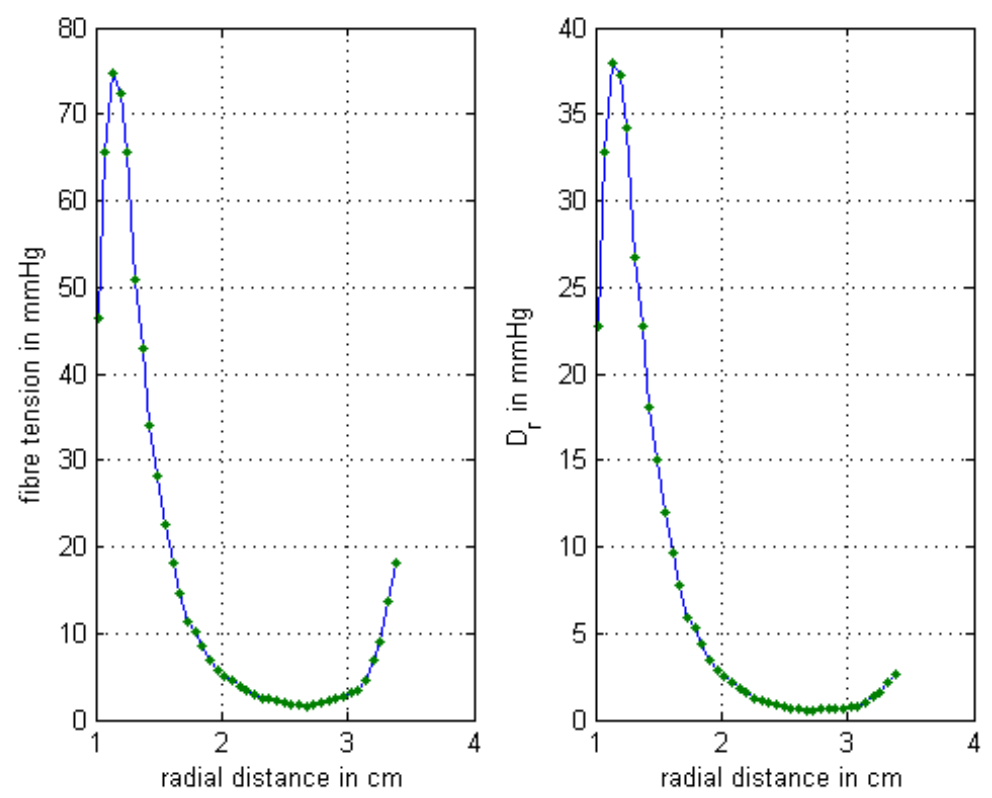

Figure 2: Radial variation from endocardium to epicardium of the fibre tension $T$ reproduced from [7] (left), and of the radial active force/unit volume of the myocardium $D_{r}$ (right).

shows the variation of $D_{r}$ calculated from eqn (22), part one and (39). Similarly the active stress components $q_{z \theta}$ and $q_{z z}$ are calculated respectively from eqn (22), parts two and three, $\sigma_{z \theta}$ and $\sigma_{z z}$ are calculated respectively from eqns (27) and (28) and shown in fig. 3. We took the ventricular pressure $P_{i}=10 \mathrm{mmHg}$ and the epicardial pressure $P_{o}=0 \mathrm{mmHg}$ in the calculation of $\tau_{a v}$ from eqn (29). The two quantities $d \chi / d r$ and $d \omega / d R$ are small and have be neglected in the calculation that follows. Consequently from eqns (35) - (38) one can derive the following equation to calculate $\sigma_{r r}$

$$
\frac{\sigma_{z \theta}}{\sigma_{z z}-\sigma_{r r}}=\frac{\left(k_{\theta} \alpha r\right) / R^{2}+k_{z} \psi r}{k_{\theta}^{2} / R^{2}+k_{z}^{2}-R^{2} /(K r)^{2}}
$$

and the following equation to calculate $\sigma_{\theta \theta}$

$$
\frac{\sigma_{z \theta}}{\sigma_{\theta \theta}-\sigma_{r r}}=\frac{\left(k_{\theta} \alpha r\right) / R^{2}+k_{z} \psi r}{(\alpha r)^{2} / R^{2}+(\psi r)^{2}-R^{2} /(K r)^{2}}
$$

The radial variation of $\sigma_{r r}$ and $\sigma_{\theta \theta}$ is shown respectively in the left and right side of fig. 4. 

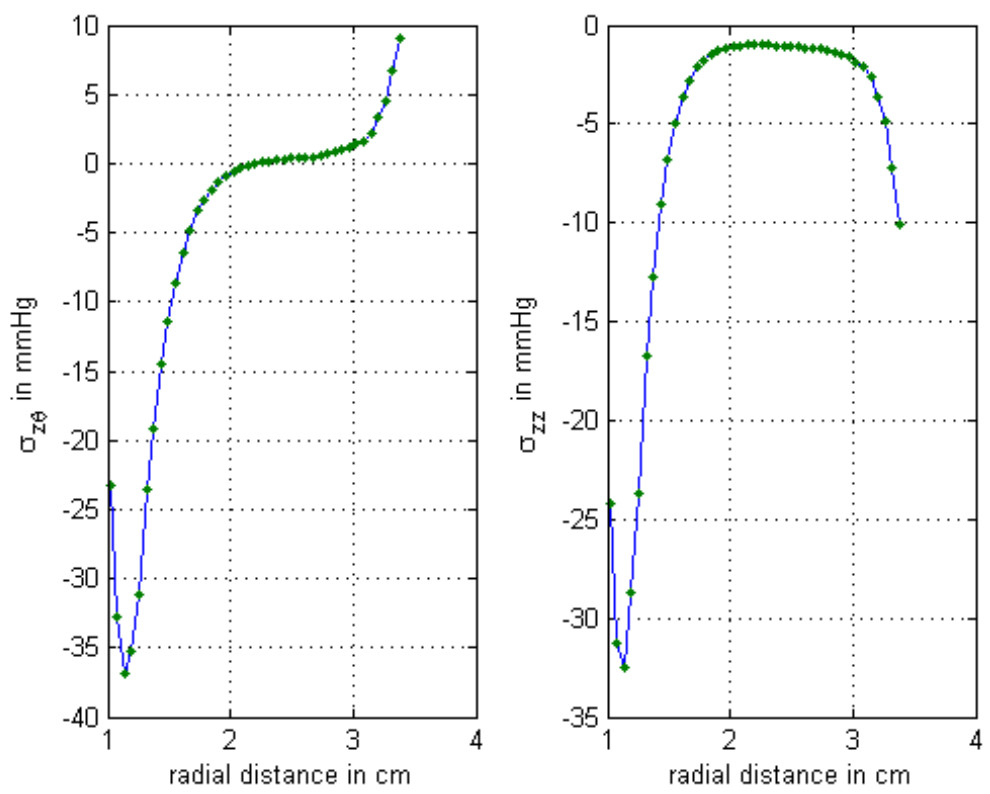

Figure 3: Radial variation from endocardium to epicardium of the stress $\sigma_{z \theta}$ (left), and of the axial stress $\sigma_{z z}$ (right).
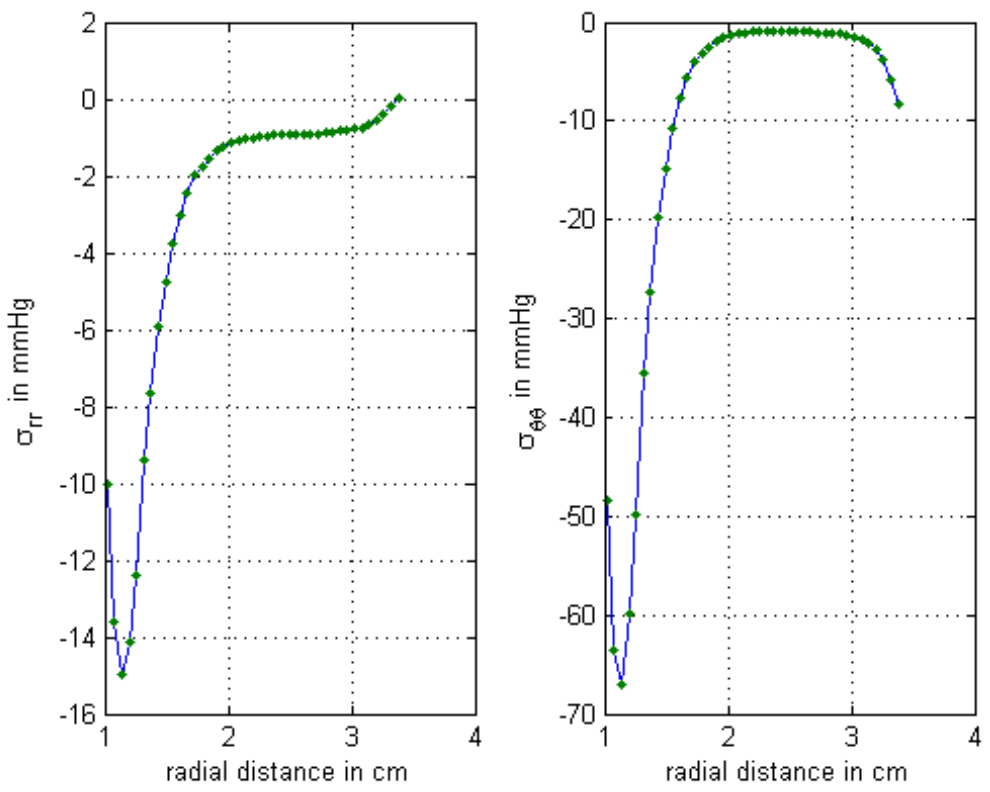

Figure 4: Radial variation from endocardium to epicardium of the radial stress $\sigma_{r r}$ (left), and of the circumferential stress $\sigma_{\theta \theta}$ (right). 


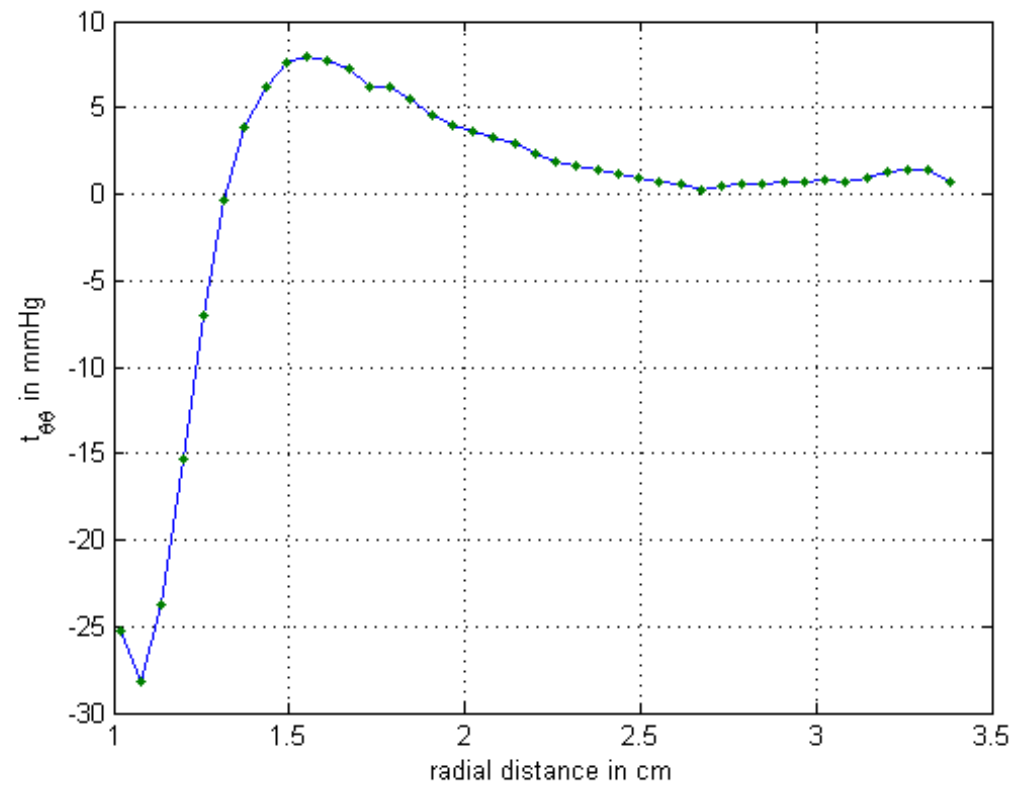

Figure 5: Radial variation of the total circumferential stress $t_{\theta \theta}$ from endocardium to epicardium.

In the calculation of $\sigma_{r r}$ and $\sigma_{\theta \theta}$ we have made use of the two following conditions, first the incompressibility condition

$$
\alpha k_{z}-\psi k_{\theta}=K
$$

The second condition is that numerator of eqn (41) for $\sigma_{z \theta}$ is zero for $r_{\text {zero }}=2.2 \mathrm{~cm}$ from fig. $3\left(R_{\text {zero }}^{2}=5.1160\right.$ from eqn (11), part two), which gives

$$
\alpha k_{\theta}+\psi k_{z} R_{\text {zero }}^{2}=0
$$

Eqns (42) and (43) are solved to express $\alpha$ and $\psi$ in terms of $k_{z}$ and $k_{\theta}$ as follows

$$
\begin{gathered}
\psi=-\frac{k_{\theta} K}{k_{\theta}^{2}+k_{z}^{2} R_{\text {zero }}^{2}} \\
\alpha=\frac{k_{z} K R_{\text {zero }}^{2}}{k_{\theta}^{2}+k_{z}^{2} R_{\text {zero }}^{2}}
\end{gathered}
$$

These values of $\psi$ and $\alpha$ are substituted into eqn (40) evaluated at $r_{i}\left(\sigma_{r r}=\right.$ $\left.P_{i}=10 \mathrm{mmHg}\right)$ and at $r_{o}\left(\sigma_{r r}=-P_{o}=0\right)$ with $\sigma_{z z}$ calculated from eqn (28). The two equations obtained in this way are solved by using the Newton iteration 
algorithm to calculate the two roots $k_{\theta}$ and $k_{z}$ of a system of two coupled equations. For $r_{i}=1.02 \mathrm{~cm}, r_{o}=3.38 \mathrm{~cm}, K=1.028, R_{i}=1.1 \mathrm{~cm}, R_{o}=3.4474$ $\mathrm{cm}$, we have calculated $k_{\theta}=-0.1472, k_{z}=1.0043$, eqns (44) and (45) give $\alpha=$ 1.0228 and $\psi=0.0057$. These are the values used to calculate the results of fig. 4.

From the results shown in figs 3 to 6 , the radial variation of each of the stress components appears to be similar to that reported in ref. [9] with a difference of sign probably due to the fact that we use the convention that a negative stress represents compression, a positive stress represents tension. It is also important to note the difference between the stress components $\sigma_{i j}$ and $t_{i j}$ as is clear for instance by comparing fig. 4 (right) and fig. 5, and also how the three quantities $W_{l}, W_{\lambda N}$ and $D_{r}$ can be expressed directly in terms of the muscular fibre $T$.
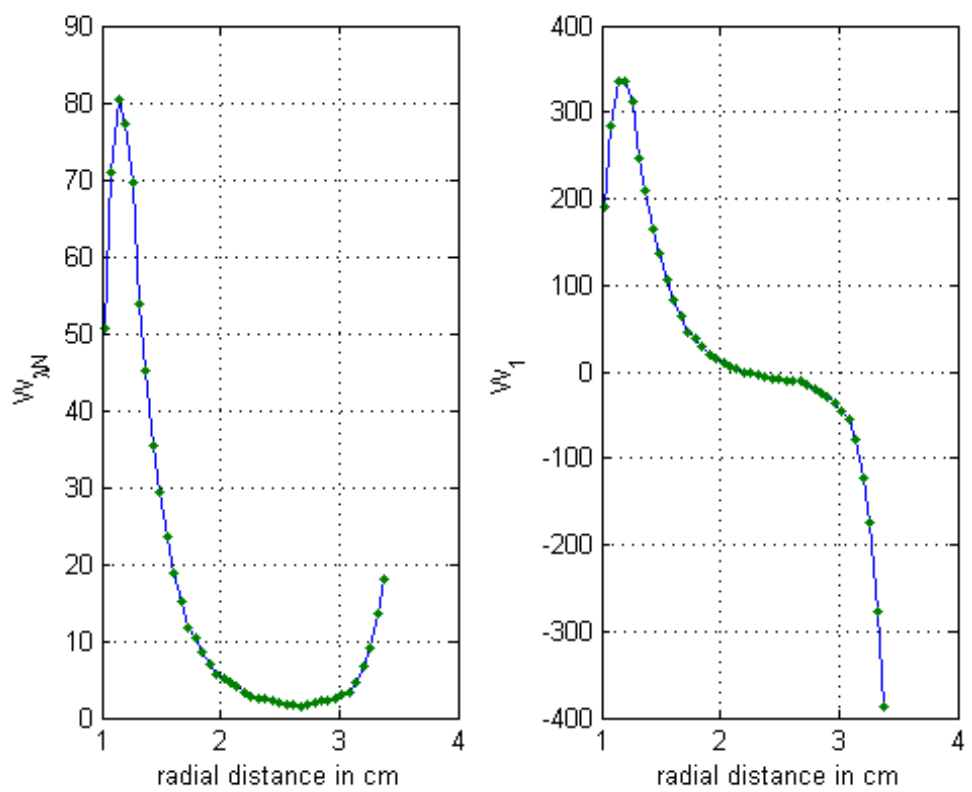

Figure 6: Radial variation from endocardium to epicardium of $W_{\lambda N}$ (eqn (31)) (left), and of $W_{l}$ (eqn (38)) (right).

\section{Conclusion}

By introducing the concept of radial active force/unit volume of the myocardium, we have shown that it is possible to calculate the stress induced in the passive medium of the myocardium and the components of the active stress generated by the muscular fibre. One should note that all the calculations have been carried out without having to assume a model for the pseudo strain energy function; instead knowledge of the muscular fibre stress generated in the direction of the muscular fibre was necessary for our calculation. It is also 
evident that the trend in the literature to split the pseudo strain energy function into an isotropic and a directional component is equivalent to the splitting of the total stress into two components as explained in this study.

\section{References}

[1] Shoucri, R.M., The pressure-volume relation and the mechanics of left ventricular contraction, Japanese Heart Journal, 31, pp. 713-729, 1990.

[2] Shoucri, R.M., Theoretical study of pressure-volume relation in left ventricle, American Journal of Physiology, 260, pp. H282-H291, 1991.

[3] Shoucri, R.M., Active and passive stresses in the myocardium, American Journal of Physiology, 279, pp. H2519-H2528, 2000.

[4] Shoucri, R.M., The calculation of the intramyocardial stress, Technology and health Care, 10, pp. 11-22, 2002.

[5] Shoucri, R.M., Studying the mechanics of left ventricular contraction, IEEE engineering in Medicine and Biology Magazine, 17, pp. 95-101, May/June 1998.

[6] Shoucri, R.M., Comparison between linear elasticity and large elastic deformation in the study of the contraction of the myocardium, Modelling in Medicine and Biology VII, ed. C.A. Brebbia, WIT Press: Southampton \& Boston, pp. 3-13, 2007.

[7] Feit, T.S., Diastolic pressure-volume relations and distribution of pressure and fiber extension across the wall of a model left ventricle, Biophysical Journal, 28, pp. 143-166, 1979.

[8] Nevo, E. \& Lanir, Y., Structural finite deformation model of the left ventricle during diastole and systole, Journal of Biomechanical Engineering, 111, pp. 342-349, 1989.

[9] Humphrey, J.D. \& Yin, F.C.P., Constitutive relations and finite deformations of passive cardiac tissue II: stress analysis in the left ventricle, Circulation Research, 65, pp. 805-817, 1989.

[10] Guccione, J.M., McCulloch, A.D. \& Waldman, L.K., Passive material properties of intact ventricular myocardium determined from a cylindrical model, Journal of Biomechanical Engineering, 113, pp. 42-45, 1991.

[11] Spencer, A.J.M., Deformation of fiber-reinforced Materials, Clarendon Press: Oxford, U.K., p. 82, 1972.

[12] Arts, T., Bovendeerd, P.H.M., Prinzen, F.W. \& Reneman, R.S., Relation between left ventricular cavity pressure and volume and systolic fiber stress and strain in the wall, Biophysical Journal, 59, 93-102, 1991.

[13] Driessen N.J.B., Bouten C.V.C. \& Baaijens F.P.T., A structural constitutive model for collagenous cardiovascular tissues incorporating the angular fiber distribution, Journal of Biomechanical Engineering, 127, 494-503, 2005.

[14] Zulliger M.A., Fridez P., Hayashi K. \& Stergiopoulos N., A strain energy function for arteries accounting for wall composition and structure, Journal of Biomechanics, 37, 989-1000, 2004.

[15] Chadwick, R.S., Mechanics of the left ventricle, Biophysical Journal, 39, 279-288, 1982. 